\title{
Una propuesta de marco conceptual para el desarrollo de proyectos de investigación en Entomología y ciencias afines
}

\author{
A proposal of a conceptual framework for the development of entomology research projects and related sciences
}

\section{ALEJANDRO G. FARJI-BRENER ${ }^{1}$}

\begin{abstract}
${ }^{1}$ Doctor en Cs. Biológicas. Laboratorio de Investigaciones en Hormigas (LIHO) - INIBIOMA-CONICET, Bariloche, Argentina. Centro Universitario Bariloche, Universidad Nacional del Comahue. Pasaje Gutiérrez 1125, (8400) Bariloche, Argentina, alefarji@yahoo.com, https://orcid.org/0000-0001-72513866
\end{abstract}

\begin{abstract}
Autor para correspondencia. Alejandro G. Farji-Brener. Doctor en Cs. Biológicas. Laboratorio de Investigaciones en Hormigas (LIHO) - INIBIOMA-CONICET y Centro Universitario Bariloche, Universidad Nacional del Comahue. Pasaje Gutiérrez 1125, (8400) Bariloche, Argentina, alefarji@yahoo.com, https://orcid. org/0000-0001-7251-3866.
\end{abstract}

Citación sugerida / Suggested citation: FARJI-BRENER, A. G. 2019. Una propuesta de marco conceptual para el desarrollo de proyectos de investigación en Entomología y ciencias afines. Revista Colombiana de Entomología 45 (1): e7805 https://doi.org/10.25100/socolen. v45i1.7805

Recibido: 31-may-2018

Aceptado: 10-oct-2018

Publicado: 30-ago-2019

Revista Colombiana de Entomología ISSN (Impreso): 0120-0488

ISSN (En línea): 2665-4385

http://revistacolombianaentomologia.univalle.edu.co/

Open access

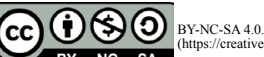

Publicadores / Publishers:

Sociedad Colombiana de Entomología

SOCOLEN (Bogotá, D. C., Colombia)

http://www.socolen.org.co

Universidad del Valle (Cali, Colombia)

http://www.univalle.edu.co/

(C) 2019 Sociedad Colombiana de Entomología - SOCOLEN y Universidad del Valle - Univalle
Resumen: La planificación de proyectos de investigación en Entomología es un desafío que muchos estudiantes no superan con éxito. La falta de entrenamiento formal sobre cómo desarrollar proyectos, la excesiva importancia a los análisis en vez de a las ideas y la ausencia de un marco conceptual sencillo son algunas de las posibles causas de este problema. En este ensayo se describe y discute un marco conceptual de utilidad didáctica para guiar a los estudiantes en la planificación de sus proyectos entomológicos y de otras ciencias afines. El mismo consta de tres aproximaciones y tres enfoques. Las aproximaciones propuestas son la descriptiva, deductiva observacional y deductiva experimental. La primera sirve para plantear proyectos descriptivos, en donde se descubren y describen patrones y, por ende, no es necesario proponer hipótesis previas ni hacer predicciones. Las aproximaciones deductivas emplean el método hipotético-deductivo, en donde se plantean ideas previas y sus posibles consecuencias si éstas son verdaderas; pero en el primer caso se predicen resultados provenientes de observaciones y en el segundo de manipulaciones. Se plantean tres enfoques; el comparativo, correlativo y el manipulativo. Los primeros dos enfoques pueden emplearse tanto en las aproximaciones descriptivas como deductivas observacionales, pero las manipulaciones son imprescindibles para la aproximación experimental. Todas estas aproximaciones y enfoques poseen tanto fortalezas como debilidades, son complementarias más que excluyentes y son ilustradas en el texto con un ejemplo concreto. Se espera que este marco conceptual, por su simpleza y valor didáctico, sea una herramienta pedagógica útil y práctica para docentes y estudiantes.

Palabras clave: Estudios descriptivos y deductivos, epistemología, educación universitaria, investigación científica.

Abstract: Planning research projects is a challenge that many students fail to successfully overcome. A lack of formal training in research planning and design, an excessive emphasis on analysis rather than on ideas and a lack of a guide are some of the possible causes of this problem. This essay proposes a conceptual framework that is of didactic utility to guide students in their research projects in entomology and related sciences. Three approximations and three approaches are presented. The proposed approximations are: descriptive, deductive observational and deductive experimental. The first is useful to design descriptive projects, where natural patterns are discovered and determined, and thus it is unnecessary and inaccurate to specify hypotheses and predictions. Deductive approximations use the hypothetical-deductive method and thus need to specify a priori the ideas being tested and the consequences if they are true. In the first deductive approximation, the predictions come from observational data, while in the second they come from experiments. Three approaches proposed are: comparative, associative and manipulative. The first two approaches can be used in both the descriptive and the deductive observational approximations. However, the manipulative approach is necessary if the experimental approximation is used. All these approximations and approaches have strengths and weaknesses; they are complementary rather than exclusive and are illustrated with an ecological study. It is expected that this conceptual framework may be a didactic and useful tool for teachers and students.

Keywords: Descriptive and deductive studies, epistemology, university education, scientific research.

\section{Introducción}

El desarrollo conceptual, la planificación y la puesta en práctica de proyectos de investigación en Entomología y ciencias afines es un desafío que muchos estudiantes de grado y postgrado no superan con éxito. Es común que los estudiantes posean dificultades para formular preguntas, encuadrarlas en marcos conceptuales, definir hipótesis, deducir sus predicciones y planificar un diseño adecuado para responder sus inquietudes. Las posibles causas de este fenómeno incluyen desde la 
falta de entrenamiento formal en la adquisición del conocimiento como parte de la tarea de resolver problemas (Fukami 2013), hasta directores que no estimulan la creatividad y capacidad crítica de sus estudiantes (Farji-Brener 2007; Lee et al. 2007). Además, en muchas facultades la obtención del grado no requiere la realización de un trabajo de tesis, con lo cual los estudiantes se enfrentan por primera vez al desafío de desarrollar un proyecto de investigación cuando deben planificar su maestría o doctorado. Metafóricamente hablando, es como salir a correr una maratón de 25 kilómetros sin siquiera haber entrenado para los 1.000 metros. Sin embargo, entre todas las causas anteriormente descritas, la ausencia de un marco conceptual amplio, sencillo y didáctico es quizás una de los obstáculos más relevantes para que los estudiantes planifiquen adecuadamente sus proyectos de investigación.

\section{Sé $R$ o no sé R: ¿esa es la cuestión?}

Toda construcción, para ser estable, necesita ser edificada desde su base. Esto no solo es válido para la arquitectura sino también para la construcción del conocimiento. Pero paradójicamente, la educación formal se ha enfocado más en mejorar los pisos superiores dejando a los cimientos desatendidos. Los cursos de estadística y de diseño experimental están incorporados a la educación formal universitaria desde hace tiempo, pero se sigue enfatizando sobre los análisis de datos, los modelos jerárquicos y el tráfico de secuencias de comandos de computación de R ("scripts") en vez de discutir las ideas conceptuales que sustentan los proyectos. Un diseño experimental complejo y un análisis estadístico sofisticado no aseguran la calidad de una investigación, ya que ambos son solo una etapa de las muchas existentes en el proceso de adquirir conocimiento (Hurlbert 1984). Cómo plantear adecuadamente una pregunta de interés sigue siendo el pilar $\mathrm{y}$, a la vez, la mayor dificultad para los estudiantes que comienzan su carrera académica en investigación (Farji-Brener 2003; Feinsinger 2013). Lamentablemente, son escasas las materias universitarias que enfatizan sobre esta dificultad. En este ensayo se describirá y discutirá un marco conceptual que propone aproximaciones $\mathrm{y}$ enfoques de utilidad didáctica para guiar a los estudiantes en la planificación de sus proyectos en Entomología y ramas afines. Estas modalidades no son, en sí mismas, originales ya que han sido descritas con diferentes nombres o discutidas por separado en otros trabajos (ver, por ejemplo, Lakatos 1983; Bunge 1997; Ford 2000; Farji-Brener 2003; Marone y Galetto 2011; Feinsinger 2013). Sin embargo, la integración conceptual y la definición de conceptos aquí propuestas pueden ser herramientas didácticas útiles para docentes y estudiantes.

\section{Aproximaciones para el desarrollo de proyectos entomológicos: el dilema de plantear o no hipótesis}

Hace casi 15 años escribí un artículo de discusión sobre el método hipotético-deductivo que tuvo efectos inesperados (Farji-Brener 2003). Allí se discutían las diferencias conceptuales entre los términos "hipótesis" y "predicciones", comúnmente mal empleados. Ese artículo, y otros que le sucedieron (Farji-Brener 2004; 2006; 2007; 2009) me pusieron en un papel originalmente no buscado: el de defensor del uso obligatorio de las hipótesis en los proyectos de investigación. Como consecuencia, durante mucho tiempo he sido testigo cómo los estudiantes con proyectos de investigación carentes de hipótesis trataban a posteriori de incluir forzadamente di- cho término (con pésimos resultados) y he sido asociado con autores que confieso jamás he leído. Sin embargo, no todas las investigaciones necesitan la formulación de hipótesis previas.

No existe una única manera de hacer investigación científica. En este ensayo se proponen dos aproximaciones que determinan diferentes tipos de proyectos: aquellos que pretenden describir patrones y aquellos que desean comprender los mecanismos que lo generan. Cuando el objetivo es verificar un patrón o describir su variación espacial o temporal, no es necesario proponer hipótesis a priori. Estamos frente a un estudio descriptivo, en donde no se pretende conocer los procesos detrás del patrón sino solo verificar su existencia y describirlo. Como es lógico, tampoco se plantean "resultados esperados" o predicciones, porque al no poner a prueba una hipótesis el investigador no "espera" ningún resultado en particular, solo describe lo que encuentra. Este tipo de investigaciones es fundamental para el avance de la ciencia: sin la confirmación de patrones y su descripción no se podría poner a prueba sus eventuales causas (Lawton 1996). Los estudios descriptivos son la base sobre la cual se plantean las hipótesis, y la verificación de patrones es la antesala para descartar el azar como mecanismo. En este tipo de estudios, las hipótesis se plantean a posteriori del trabajo, como posibles explicaciones a los patrones encontrados. Es por eso que la sección de "discusión" en los trabajos descriptivos generalmente se basa en el planteo de hipótesis para intentar explicar los resultados hallados, las cuales no son puestas a prueba en dicho estudio, sino que quedan como posibles opciones a ser exploradas en un futuro. La fortaleza de esta aproximación es que confirma patrones y es la fuente para proponer hipótesis en futuros estudios. Su debilidad es que el mecanismo que genera el patrón queda, transitoriamente, en la incertidumbre.

Por otra parte, los estudios que pretenden conocer las causas de un patrón deben proponer hipótesis; esto es deben formular diferentes mecanismos posibles y deducir sus resultados esperados si éstos son verdaderos (o sea, formular predicciones). Esto es indispensable porque las ideas solo se pueden poner a prueba indirectamente, evaluando sus consecuencias (Popper 1959; Bunge 1997; Farji-Brener 2003; Marone y Galetto 2011). En el mejor de los mundos, y en la más completa acepción del método hipotético-deductivo, se deberían poner a prueba diferentes hipótesis para un mismo patrón. De esta manera, confrontando los resultados con las predicciones de cada hipótesis, podremos descartar la(s) más improbable(s) y quedarnos con la "transitoriamente" verdadera (Popper 1959; Bunge 1997). En esta instancia los proyectos pueden tomar dos rumbos, dependiendo del empleo o no de experimentos. Si el estudio, por razones de temática, escala, presupuesto o logística no contempla la realización de experimentos, los resultados esperados son formulados y buscados en forma de patrones ya existentes en la naturaleza. Esta aproximación se denominará "deductivo observacional", porque implica el planteo de hipótesis, la deducción de sus consecuencias, y la comparación de los resultados esperados con aquellos provenientes de observaciones. Si el estudio contempla la realización de experimentos, se denominará "deductivo experimental", y la única diferencia conceptual con la aproximación anterior es que las predicciones son formuladas como resultados esperados que provienen de manipulaciones. En los trabajos deductivos observacionales, los patrones propuestos como resultados esperados (i. e., predicciones) pueden ser consistentes con los finalmente obtenidos, pero sin la realización de experimentos será difícil confirmar fehacientemente la 


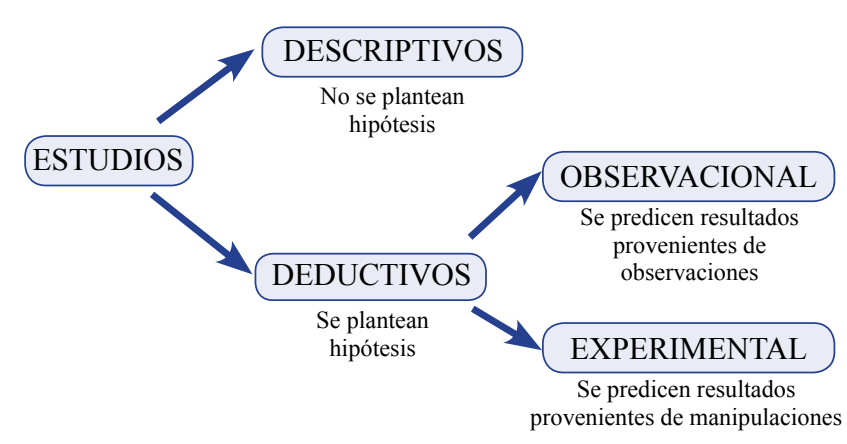

Figura 1. Esquema del marco conceptual propuesto, en donde existen tres aproximaciones para realizar proyectos de investigación: la descriptiva, la deductiva observacional y la deductiva experimental.

existencia de los mecanismos propuestos. En el caso de los estudios deductivos experimentales, la realización de experimentos permite al investigador poner a prueba de forma más sólida los mecanismos propuestos, porque puede manipular las variables de interés. Ambas aproximaciones son un buen ejercicio intelectual, porque obligan al investigador a ejercer su capacidad deductiva.

En consecuencia, un proyecto de investigación puede ser de índole descriptivo, en donde no es necesario el planteo de hipótesis ni predicciones a priori, o deductivo (sea observacional o experimental), en donde se deben plantear las hipótesis a priori que se supone generan el patrón y sus resultados esperados si esas ideas son correctas (Fig. 1). Todas estas aproximaciones son valiosas para el avance de la Entomología y pueden ser complementarias. Por ejemplo, si no se conoce un sistema, lo ideal es que primero se explore el mismo con una aproximación descriptiva, en donde se respondan preguntas sencillas de historia natural. Luego, con los patrones ya descritos, se pueden formular diferentes hipótesis sobre sus causas, las cuales podremos poner a prueba con nuevas observaciones o experimentos. Finalmente, la elección de estas diferentes aproximaciones dependerá del tipo de pregunta, la curiosidad del investigador, conocimiento previo de la historia natural del sistema/organismo en estudio y de las posibilidades logísticas o económicas existentes. Un proyecto entomológico puede simplemente describir la variación en riqueza de especies de escarabajos a lo largo de un gradiente ambiental sin proponer hipótesis previas, sino objetivos o preguntas. O se puede plantear el mismo proyecto pero poniendo a prueba ideas basadas en marcos conceptuales existentes (i.e., hipótesis de la complejidad estructural, o hipótesis de la productividad, etc.), los cuales predicen cómo deberían variar la riqueza de especies de escarabajos (y otros insectos) en un gradiente. La diferencia es que la planificación previa de la puesta a prueba de hipótesis específicas permite diseñar la investigación de una manera más eficaz, incluyendo la planificación de experimentos si éstos son factibles.

\section{Enfoques para el desarrollo de proyectos entomológicos: comparar, asociar, manipular}

Por otra parte, también existen tres posibles enfoques para responder a los objetivos del proyecto: el comparativo, el correlativo y el manipulativo. El comparativo simplemente compara la variable de interés entre dos o más situaciones. El correlativo analiza cómo la variable de interés cambia a lo largo de un gradiente (esto es, cuando cambian otras varia- bles). Finalmente, el manipulativo se centra en la realización de experimentos (cuya naturaleza puede ser comparativa o correlativa) y el análisis de sus resultados. Nuevamente, el empleo de uno u otro enfoque va a depender de la pregunta, el sistema en estudio, la creatividad del investigador, el tiempo, y los recursos logísticos/económicos existentes. En un mundo (y proyecto) ideal, se pueden emplear todos los enfoques, ya que metafóricamente hablando, éstos representan las "patas" de la mesa de las ideas: cuantas más "patas" existan, accederemos a más chances de sostener (o descartar) las hipótesis propuestas. La ventaja de conocer a priori el enfoque que se va a emplear es que se puede planificar mejor el diseño y los análisis, ganando eficiencia en el desarrollo del proyecto (ver un ejemplo más adelante). Además, el conocimiento a priori de la existencia de estos enfoques es un estímulo en sí mismo para, al momento de planificar la investigación, confirmar si estamos empleando todos las herramientas que podemos/debemos usar para responder la pregunta de interés.

\section{La conexión entre aproximaciones y enfoques: un ejemplo}

Estos enfoques y las aproximaciones anteriormente descritas están íntimamente asociados. Por ejemplo, las mediciones comparativas o correlativas pueden emplearse tanto en estudios descriptivos como en aquellos deductivos observacionales; pero el enfoque manipulativo es fundamental en estudios con una aproximación experimental. Para ilustrar estos conceptos detallaré su aplicación a un caso concreto. Supongamos que observamos en el campo que cierta especie de hormiga se encuentra más frecuentemente en cierta especie de planta. La primera aproximación podría ser de índole descriptiva, donde simplemente nos plantearíamos la pregunta " ¿Se asocia la especie de hormiga X con la especie de planta Y?" o propondríamos el objetivo de "describir la existencia de asociación entre la especie de hormiga X con la especie de planta Y". Este proyecto es claramente descriptivo, no pretende poner a prueba ninguna hipótesis previa y por lo tanto no se espera ningún resultado en particular. Forzar este estudio descriptivo a uno hipotético-deductivo nos "obliga" a plantear hipótesis engañosas y predicciones circulares como "Si la especie de hormiga X se asocia a la planta $Y$, espero una mayor frecuencia de la especie de hormiga $\mathrm{X}$ con la especie de la planta $\mathrm{Y}$ que con otras especies de plantas". Nótese que, pese a la lógica del pensamiento, la predicción es simplemente la definición de asociación y no se pone a prueba ningún mecanismo, valor adaptativo para alguna de las partes, o causa de dicho patrón. O sea, estamos cayendo en un enunciado circular empleando de manera errónea los términos hipótesis y predicciones (Farji-Brener 2003). Una vez confirmada la asociación, se pueden proponer hipótesis que traten de explicar su causa o el valor adaptativo para alguno de sus componentes. En esta instancia es fundamental entrenarse en enunciar las hipótesis completas (incluyendo el patrón sobre el cual se inspiran) tanto como reflexionar sobre la palabra "clave" que representa el mecanismo puesto a prueba. Una opción puede ser la hipótesis de la protección, en donde las hormigas poseen sus nidos en la planta y la "defienden" contra insectos herbívoros. En este caso, la hipótesis completa puede enunciarse de la siguiente manera: "la especie de planta $\mathrm{Y}$ se asocia a la especie de hormiga $\mathrm{X}$ porque esta hormiga la protege contra sus enemigos naturales". La palabra "clave" en esta hipótesis es protección. Nótese que esta idea es una posibilidad dentro de muchas otras (por ejemplo, la planta puede 
estar asociada a las hormigas porque éstas le proveen cierto nutriente en particular, palabra "clave"; nutrición). La hipótesis de la protección puede tener varias predicciones relacionadas con el nivel de daño foliar de la planta hospedera, y nos permite planificar proyectos realizando o no manipulaciones (o sea, mediante las aproximaciones deductiva observacional y experimental, respectivamente). Si no se tiene el tiempo, los ayudantes, el dinero para contratar técnicos o adquirir equipo, o la logística para realizar experimentos, se pueden emplear los enfoques comparativo y/o correlativo. Por ejemplo, si las hormigas defienden a las plantas de sus enemigos naturales, se puede predecir que plantas con hormigas tendrán menos herbivoría que plantas sin hormigas (enfoque comparativo) o que el nivel de daño foliar se reducirá a medida que aumente el número de hormigas por planta (enfoque correlativo). Es interesante destacar que ambos enfoques pueden estar íntimamente asociados: las medidas en el enfoque comparativo pueden ser empleadas en el enfoque correlativo si el diseño se encuentra bien planeado (Fig. 2). Si a priori se planificaron ambos enfoques sabemos que en el campo debemos estimar el número de hormigas por planta, variable fundamental para la predicción correlativa (aunque no para la predicción comparativa). Si no se tuvo en cuenta ese detalle, no se podrá emplear el enfoque correlativo o se deberá volver al campo para recolectar esos datos. Por otra parte, si se puede excluir o adicionar colonias de hormigas se podrá plantear un proyecto con una aproximación experimental, la cual requiere del enfoque manipulativo (Fig. 2). Moraleja: el conocimiento a priori de la aproximación y los enfoques a emplear permiten maximizar el esfuerzo de muestreo y realizar un diseño más adecuado para responder las preguntas de interés.

En resumen, para desarrollar un proyecto de investigación en Entomología (y ciencias afines) se propone la existencia de tres aproximaciones (una de las cuales no requiere proponer hipótesis previas), y tres posibles enfoques. El empleo tanto de estas aproximaciones como de los diferentes enfoques va a depender del conocimiento de la historia natural, curiosidad, logística y recursos financieros del investigador, entre otros factores. Todas estas aproximaciones y enfoques poseen virtudes y defectos (Tabla 1). Como se mencionó, la aproximación descriptiva es vital para desentrañar patrones, base fundamental para proponer hipótesis y avanzar así en el conocimiento de
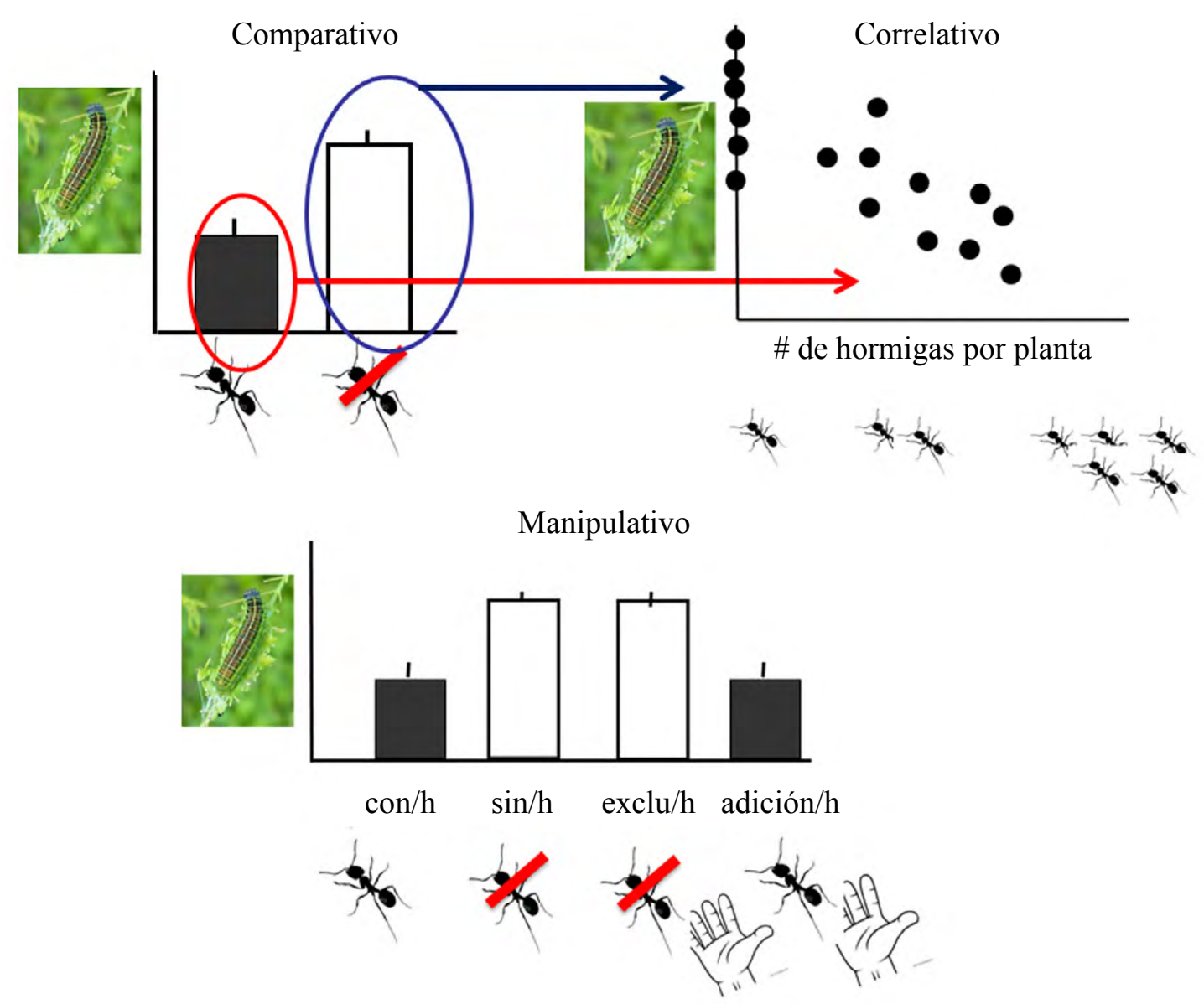

Figura 2. Diferentes enfoques mediante los cuales podemos responder preguntas de investigación en Entomología y otras ciencias afines. El enfoque comparativo compara dos o más situaciones, el correlativo mide cambios en una variable de interés a lo largo de un gradiente, y el enfoque manipulativo requiere de experimentación. En este caso, se representan ejemplos de los 3 enfoques para poner a prueba la hipótesis de que las hormigas protegen a su planta hospedera de los herbívoros. Las flechas que unen el enfoque comparativo y correlativo resaltan su relación; los mismos datos pueden emplearse para ambos enfoques. Los tratamientos del enfoque manipulativo están representados por una mano que excluye y adiciona hormigas a plantas que originalmente poseen y carecen de ellas, respectivamente. En todos los casos se grafican los resultados esperados si las hormigas protegen a las plantas. Las barras de error son figurativas y solo ilustran la necesidad de emplear verdaderas réplicas. 
Tabla 1. Descripción de diferentes aproximaciones y enfoques para desarrollar proyectos de investigación en Entomología y ciencias afines. Se describe las características de cada tipo de proyecto ilustrado con un ejemplo.

\begin{tabular}{|c|c|c|c|c|c|c|}
\hline $\begin{array}{l}\text { Tipos de } \\
\text { proyecto }\end{array}$ & Características & Enfoques & $\begin{array}{c}\text { Hipótesis y } \\
\text { predicciones }\end{array}$ & Fortalezas & Debilidades & Ejemplo \\
\hline Descriptivo & $\begin{array}{l}\text { Verifica y descri- } \\
\text { be patrones. }\end{array}$ & $\begin{array}{c}\text { Comparativo, } \\
\text { Correlativo }\end{array}$ & $\begin{array}{l}\text { No hay. Al no } \\
\text { proponer hipótesis } \\
\text { previas no hay re- } \\
\text { sultados esperados }\end{array}$ & $\begin{array}{l}\text { Determina la exis- } \\
\text { tencia de patrones, } \\
\text { base necesaria para } \\
\text { proponer hipótesis }\end{array}$ & $\begin{array}{c}\text { No indaga sobre las } \\
\text { posibles causas del } \\
\text { patrón }\end{array}$ & $\begin{array}{l}\text { Confirmar la asociación } \\
\text { entre cierta hormiga y } \\
\text { una especie de planta, } \\
\text { describir variaciones en } \\
\text { gradientes. }\end{array}$ \\
\hline $\begin{array}{l}\text { Deductivo } \\
\text { observacional }\end{array}$ & $\begin{array}{l}\text { Propone mecanis- } \\
\text { mos (hipótesis) } \\
\text { para explicar pa- } \\
\text { trones y los pone } \\
\text { a prueba mediante } \\
\text { predicciones que } \\
\text { no provienen de } \\
\text { experimentos. }\end{array}$ & $\begin{array}{c}\text { Comparativo, } \\
\text { Correlativo }\end{array}$ & $\begin{array}{l}\text { Se proponen hipó- } \\
\text { tesis y se evalúan } \\
\text { los resultados } \\
\text { esperados si dichas } \\
\text { ideas son correc- } \\
\text { tas, buscando } \\
\text { patrones diferentes } \\
\text { al patrón que dio } \\
\text { origen a la idea. }\end{array}$ & $\begin{array}{c}\text { Genera evidencias } \\
\text { que pueden ser o no } \\
\text { consistentes con la } \\
\text { hipótesis. Descarta } \\
\text { ideas incorrectas. } \\
\text { Son un buen ejercicio } \\
\text { intelectual. }\end{array}$ & $\begin{array}{c}\text { Al no existir } \\
\text { experimentación, } \\
\text { sus resultados son } \\
\text { asociaciones que no } \\
\text { terminan de con- } \\
\text { firmar las hipótesis } \\
\text { propuestas. }\end{array}$ & $\begin{array}{c}\text { Si las hormigas } \\
\text { defienden a su planta } \\
\text { hospedera de herbívoros, } \\
\text { se espera que plantas } \\
\text { con hormigas posean } \\
\text { menos daño foliar que } \\
\text { plantas sin hormigas, } \\
\text { o que plantas con más } \\
\text { hormigas posean menos } \\
\text { daño foliar. }\end{array}$ \\
\hline $\begin{array}{l}\text { Deductivo } \\
\text { experimental }\end{array}$ & $\begin{array}{l}\text { Propone mecanis- } \\
\text { mos (hipótesis) } \\
\text { para explicar pa- } \\
\text { trones y los pone } \\
\text { a prueba mediante } \\
\text { predicciones que } \\
\text { provienen de } \\
\text { resultados experi- } \\
\text { mentales. }\end{array}$ & Manipulativo & $\begin{array}{l}\text { Se proponen hipó- } \\
\text { tesis y se evalúan } \\
\text { sus predicciones, } \\
\text { las cuales son } \\
\text { propuestas como } \\
\text { posibles resultados } \\
\text { experimentales. }\end{array}$ & $\begin{array}{l}\text { Al venir de experi- } \\
\text { mentos “controla- } \\
\text { dos", sus resultados } \\
\text { son más sólidos para } \\
\text { apoyar o descartar las } \\
\text { hipótesis propuestas. } \\
\text { Son un buen ejercicio } \\
\text { intelectual. }\end{array}$ & $\begin{array}{l}\text { Las manipulaciones } \\
\text { pueden representar } \\
\text { situaciones poco } \\
\text { reales o generar } \\
\text { efectos indeseables. } \\
\text { Los experimentos } \\
\text { deben estar formu- } \\
\text { lados a la escala } \\
\text { correcta. } \\
\text { Para ciertas pre- } \\
\text { guntas y escalas no } \\
\text { es factible realizar } \\
\text { experimentos. }\end{array}$ & $\begin{array}{c}\text { Si las hormigas } \\
\text { defienden a su planta } \\
\text { hospedera de herbívoros, } \\
\text { se espera que plantas } \\
\text { en donde las hormigas } \\
\text { fueron excluidas experi- } \\
\text { mentalmente incremen- } \\
\text { ten su nivel de daño } \\
\text { foliar comparadas con } \\
\text { aquellas sin exclusión. }\end{array}$ \\
\hline
\end{tabular}

un organismo o sistema. Sin embargo, la mera descripción no basta para comprender un fenómeno. Por otra parte, las aproximaciones deductivas son un buen ejercicio intelectual y nos acercan más a conocer los mecanismos detrás de los patrones. Muchas veces proponer predicciones que son observaciones (o sea, nuevos patrones) es logísticamente más factible u operativo que hacer experimentos. Muchas ramas de la Entomología emplean exclusivamente la aproximación deductiva observacional por cuestiones de escala (i. e., distribución de especies en regiones o gradientes). Por otra parte, los experimentos permiten modificar la variable de interés y así comprender mejor las causas de un fenómeno, pero algunas manipulaciones son inviables de realizar por cuestiones de escala, pueden generar efectos indeseados y/o simular situaciones artificiales carentes de sentido biológico. En otras palabras, todas las aproximaciones y enfoques propuestas presentan fortalezas y debilidades; y deben considerarse complementarias más que excluyentes.

\section{Deducir e indagar a nadie le hace mal}

Los entomólogos nos dedicamos básicamente a describir y comprender las causas de la variación en los insectos (incluyendo sus comportamientos e interacciones), tanto a nivel individual como a escala de poblaciones y comunidades. Ambas tareas, describir patrones y comprender sus causas, son de fundamental importancia. Sin patrones bien descritos no se puede indagar sobre sus causas, conocimiento que sólo se puede acceder contrastando diferentes hipótesis propuestas $a$ priori y evaluando sus predicciones (Houlahan et al. 2017). En consecuencia, tanto la indagación como la deducción forman parte indisoluble de la Entomología como ciencia. En este contexto, el marco conceptual que se propone de aproximaciones y enfoques es útil para encarar estudios descriptivos como aquellos que emplean el método hipotético-deductivo, sin necesidad de una competencia infructífera entre ambas formas de acumular conocimiento. Esta propuesta brinda argumentos conceptuales adecuados para que los profesores puedan explicar con un lenguaje simple y ejemplos concretos cómo encarar una investigación en Entomología y ciencias afines. Así, los estudiantes podrán comprender fácilmente qué tipo de proyecto pretenden o pueden desarrollar. Hay varios ejercicios posibles relacionados con esta propuesta: (a) determinar las aproximaciones y enfoques empleados en trabajos ya publicados (y proponer alternativas); (b) proponer una pregunta de interés, separar a los estudiantes en grupos y que cada grupo desarrolle un proyecto bajo diferentes aproximaciones y enfoques, para luego discutir entre todos las fortalezas y debilidades de cada caso, y/o (c) realizar pequeños proyectos individuales de investigación bajo diferentes aproximaciones y enfoques. Por otra parte, se aspira que esta propuesta sirva también para que los estudiantes no sientan la imperiosa necesidad de "disfrazar" los buenos proyectos descriptivos en deductivos, forzando la inclusión de hipótesis $\mathrm{y}$ predicciones que no fueron pensadas de antemano. Dado que puede haber proyectos buenos y malos tanto descriptivos como deductivos, la ausencia de hipótesis y predicciones en trabajos, propuestas o tesis no debería relacionarse con la calidad de la investigación ni afectar las posibilidades de acceder a una beca o subsidio (Feinsinger 2013). Como se mencionó, la separación entre estudios que describen patrones y aquellos que ponen a prueba sus causas es meramente operativa y no implica juicio de valor alguno. Tanto describir patrones 
como comprender su causa es de fundamental importancia (y rima). La descripción y la deducción pueden convivir en un mundo complejo, en donde es necesaria una pluralidad de visiones para comprender mejor cómo funciona la naturaleza y resolver problemas específicos de conservación. Espero que esta propuesta se entienda como un marco conceptual amplio en donde estas visiones se complementan, y que su valor didáctico facilite la tarea de los docentes y estudiantes en el acompañamiento y desarrollo de proyectos de investigación en Entomología y otras ramas afines de la ciencia.

\section{Agradecimientos}

A.S. Amador-Vargas por dejarme robarle la idea de su bonita página de explicación de $\mathrm{R}$ (Sé $\mathrm{R}$ o no sé $\mathrm{R}$ ) para uno de mis subtítulos. Dos revisores anónimos ayudaron a mejorar versiones preliminares de este manuscrito.

\section{Literatura citada}

BUNGE, M. 1997. La ciencia, su método y su filosofía. Editorial Sudamericana, Buenos Aires. 187 p.

FARJI-BRENER, A. G. 2003. Uso correcto, parcial e incorrecto de los términos hipótesis y predicciones en ecología. Ecología Austral 13: 223-227.

FARJI-BRENER, A. G. 2004. ¿Son hipótesis las hipótesis estadísticas? Ecología Austral 14: 201-203.

FARJI-BRENER, A. G. 2006. La (significativa) importancia biológica de la no-significancia estadística. Ecología Austral 16: 79-84.

FARJI-BRENER, A. G. 2007. Una forma alternativa para la enseñanza del método hipotético-deductivo. Interciencia 32: 716720.

FARJI-BRENER, A. G. 2009. ¿Ecólogos o Ególogos? Cuando las ideas someten a los datos. Ecología Austral 19: 167-172.
FEINSINGER, P. 2013. Metodologías de investigación en ecología aplicada y básica: ¿cuál estoy siguiendo, y por qué? Revista Chilena de Historia Natural 86: 385-402. https://doi.org/10.4067/ S0716-078X2013000400002

FORD, E. D. 2000. Scientific method for ecological research. Cambridge, RU. Cambridge University Press. 588 p.

FUKAMI, T. 2013. Integrating Inquiry-Based Teaching with Faculty Research. Science 339: 1536-1537. https://doi.org/10.1126/ science. 1229850

HOULAHAN, J.; MCKINNEY, S. T.; ANDERSON, T. M.; MCGILL, B. J. 2017. The priority of prediction in ecological understanding. Oikos 126: 1-7. https://doi.org/10.1111/oik.03726

HURLBERT, S. 1984. Pseudorreplication and the design of ecological field experiments. Ecological Monographs 54: 187-211. https://doi.org/10.2307/1942661

LAKATOS, I. 1983. La metodología de los programas de investigación. Madrid: Alianza.

LAWTON, J. 1996. Patterns in ecology. Oikos 75:145-147. https:// doi.org/10.2307/3546237

LEE, A.; DENNIS, C.; CAMPBELL, P. 2007. Nature's guide for mentors. Nature 447: 791-797. https://doi.org/10.1038/447791a

MARONE, L.; GALETTO, L. 2011. El doble papel de las hipótesis en la investigación ecológica y su relación con el método hipotético-deductivo. Ecología Austral 21: 201-216.

POPPER, K. 1959. The logic of scientific discovery. Basic Books. New York. https://doi.org/10.1063/1.3060577

\section{Origen y financiación}

Este artículo fue inspirado durante el dictado de numerosos cursos de campo, en donde los estudiantes normalmente presentaban problemas para plantear y desarrollar proyectos de investigación. Pese a que no tuve fuentes de financiación especifica para escribir este artículo, su producto fue parcialmente apoyado financieramente de manera indirecta por la Organización para Estudios Tropicales (OET) y el CONICET (Argentina). 